\title{
Metodología para fortalecer la participación del personal en el Sistema de Gestión de Seguridad y Salud en el Trabajo en una empresa del sector aeronáutico*
}

\section{Methodology for strengthening the participation of personnel in Safety and Health at Work Management System in an aviation industry company}

John Alexander Gonะáles Bucurí ${ }^{* *}$

Universidad Santo Tomás

\section{RESUMEN}

Este artículo presenta los resultados de un proyecto de investigación cuyo propósito fue definir una metodología para el fortalecimiento de la participación del personal en el Sistema de Gestión de Seguridad y Salud en el Trabajo - SG-SST en una empresa del sector aeronáutico. Se diseñaron encuestas y entrevistas para evaluar el nivel de participación del personal en el SGSST y los mecanismos que los trabajadores utilizaban para involucrarse en el mismo. Se evidenció que el nivel de participación del personal de los niveles táctico y operativo fue significativo, es decir un nivel intermedio; el nivel de participación del personal directivo fue variable, entre bajo, significativo y elevado.

\footnotetext{
Artículo de resultado de investigación.

** Ingeniero industrial. Especialista en Seguridad Industrial. Magíster (c) en Calidad y Gestión Integral. Coordinador salud ocupacional en Copa Airlines Colombia. Correo electrónico: johgonzalezb@gmail.com
} 
Posteriormente, se definió una metodología que describe las acciones para fortalecer la participación del personal de los niveles estratégico, táctico y operativo de la empresa en el Sistema de Gestión de Seguridad y Salud en el Trabajo, siguiendo el ciclo de mejoramiento continuo PHVA (planear, hacer, verificar, actuar) y los mecanismos de participación información, consulta y codecisión. Expertos en seguridad y salud en el trabajo validaron la metodología propuesta, indicando que cumplió los criterios de validación definidos.

Palabras clave: participación, seguridad y salud en el trabajo, sistema de gestión, mecanismos de participación.

\section{ABSTRACT}

The purpose of this article is to present the results of a research project whose purpose was to define a methodology for strengthening the participation of personnel in the Occupational Health and Safety Management System - OHS-MS in an aeronautical sector company. Surveys and interviews were designed to assess the level of staff participation in OHS and the mechanisms workers used to become involved. It was evidenced that the level of participation of the personnel of the tactical and operative levels was significant, that is to say an intermediate level; the level of participation of the strategic staff was variable, between low, significant and high.

Subsequently, a methodology was defined that describes the actions to strengthen the participation of the personnel of the strategic, tactical and operative levels of the company in the Occupational Health and Safety Management System, following the cycle of continuous improvement PDCA (plan, do, check, act) and the mechanisms of participation information, consultation and codecision. Experts in Occupational Health and Safety validated the proposed methodology, indicating that it met the defined validation criteria.

Keywords: Participation, safety and health at work, management system, participation mechanisms.

\section{INTRODUCCIÓN}

El Sistema de Gestión de Seguridad y Salud en el Trabajo - SG-SST "debe ser liderado e implementado por el empleador con la participación de los trabajadores" (Ministerio de Trabajo, 2015), por tanto, es ineludible que el personal de los niveles operativo, táctico y estratégico sea partícipe de cada una de las etapas del SG-SST. La participación constituye un derecho y también un deber, además de ser clave para lograr el éxito del SG-SST. Con la creación del Sistema General de Riesgos Profesionales (Secretaria General de la Alcaldía Mayor de Bogotá, 1994), hoy Riesgos Laborales, se emitió una serie de normas legales destinadas a proteger la salud de los trabajadores y para mitigar los riesgos laborales generados por las empresas, no obstante "las normas legales por sí solas son insuficientes" (Organización Internacional del Trabajo - OTI, 2001).

Según el informe de Indicadores del Sistema General de Riesgos Laborales de Colombia, durante el periodo 2011 a 2015 se calificaron en promedio 637.687 accidentes de trabajo al año, equivalentes a una tasa de accidentes de 7,44 promedio anual por cada 100 afiliados al Sistema General de Riesgos Laborales; durante el mismo periodo se calificaron en promedio 9.332 enfermedades como laborales cada año, con una tasa de enfermedades calificadas de 109.39 por cada 100.000 afiliados y una tasa promedio anual de muertes calificadas como laborales de 6,41 por 100.000 (Ministerio de Trabajo de Colombia, 2016). 
Por su parte, el Informe Ejecutivo de la II Encuesta Nacional de Condiciones de Seguridad y Salud en el Trabajo en el Sistema General de Riesgos de Colombia publicado en diciembre 2013 (Ministerio de Trabajo de Colombia, 2013), reportó que tan solo el $55.9 \%$ de las empresas contaban con programa de salud ocupacional o Sistema de Gestión de Seguridad y Salud; de ese porcentaje, el $55.50 \%$ tenían panorama de factores de riesgo, $45.83 \%$ realizaban exámenes médicos, 42.68 $\%$ realizaban estudios de higiene y solo el $21.07 \%$ ejecutaban programas de vigilancia epidemiológica.

Los directivos de la empresa objeto de estudio tienen claro el compromiso con el cumplimiento de las leyes locales e internacionales: valores en acción "cumplimos siempre con las leyes y regulaciones locales e internacionales y las políticas y procedimientos de la empresa"; sin embargo, durante los últimos cinco años la tendencia de accidentalidad ha sido ascendente, se han presentado en promedio 65 accidentes de trabajo cada año, equivalentes a una tasa de 0,65 accidentes por cada 100 trabajadores; 7 enfermedades fueron calificadas como laborales, 4 enfermedades osteomusculares, 2 auditivas y 1 relacionada con el sistema nervioso central.

Según la clasificación para el análisis de causalidad de accidentes de trabajo descrita en la Norma Técnica Colombia - NTC 3701, el $53.8 \%$ de los accidentes de trabajo fueron causados por actos inseguros, $30.8 \%$ por condiciones inseguras y $15.4 \%$ por factores del trabajo. A nivel organizacional, los accidentes fueron causados por instrucción o entrenamiento deficientes, estándares deficientes de trabajo y asignación de responsabilidades poco claras (Icontec, 1995).

"La gestión de la Seguridad y Salud en el Trabajo debe ser incorporada en la gestión estratégica de las organizaciones" (Erazo, 2014), ser parte integral de la gestión de riesgos de la empresa y "puede ser integrada con otros sistemas de gestión" (OIT, 2011). A través de la gestión de riesgos se obtienen beneficios como "la administración del riesgo con enfoque proactivo en lugar de reactivo, mejorar las prácticas de seguridad, cumplimiento de los requisitos legales, cumplimiento de los objetivos organizacionales" (Mendoza y Sánchez, 2012), así como contribuir al crecimiento, rentabilidad y sostenibilidad de la organización.

La Organización Internacional del Trabajo (2011) afirma que "el empleador es el responsable de la implementación del SG-SST” (p. 1); en Colombia, el Decreto 1443 de 2014 (actualmente se encuentra dentro del Secretaría General de la Alcaldía Mayor de Bogotá Decreto 1072 de 2015) dictó las disposiciones para la implementación del SG-SST de obligatorio cumplimiento para todos los empleadores, trabajadores y centros de trabajo, incluyó diferentes acciones para la participación del personal y se definió el 1 de junio de 2017 como fecha para dar inicio a su ejecución de manera progresiva y sistemática (Secretaría General de la Alcaldía Mayor de Bogotá, 2017), esto enmarcado en el "Plan Nacional de Salud Ocupacional 2013-2021".

El proyecto de investigación tuvo como objetivo principal, definir una metodología para el fortalecimiento de la participación del personal en el Sistema de Gestión de Seguridad y Salud en el Trabajo - SG-SST en una empresa del sector aeronáutico. Como hipótesis se planteó que el nivel de participación del personal en el SG-SST de la empresa era bajo y que el mecanismo de participación que se encontraba mejor desarrollado era el de información. 


\section{Participación del personal en el Sistema de Gestión de Seguridad y Salud en el Trabajo}

La seguridad y salud en el trabajo es reconocida como:

\begin{abstract}
Aquella disciplina encaminada hacia la promoción, la protección, el mantenimiento y el mejoramiento del bienestar físico, mental y social de los trabajadores en todas sus ocupaciones (Ley 1562 de 2012), su objetivo esencial es la gestión de los riesgos en el trabajo (OIT, 2011).
\end{abstract}

Con el fin de mejorar las condiciones y el medio ambiente de trabajo, prevenir la ocurrencia de accidentes de trabajo y enfermedades laborales; también para "principalmente garantizar el bienestar y la calidad de vida de la población trabajadora" (Rodríguez y Molano, 2012), reflejado en "manifestaciones saludables" (Betancourt, 2007), como "el bienestar físico, mental y social de las personas, entre otras consecuencias positivas" (Molano y Arévalo, 2013).

Por su parte, el Sistema de Gestión de Seguridad y Salud en el Trabajo - SG-SST ${ }^{1}$, consiste en el desarrollo de un proceso lógico y por etapas, basado en la mejora continua, incluye la política, organización, planificación, aplicación, evaluación y acciones de mejora (OIT, 2001, p. 7) que tienen como objetivo proteger y promover la salud y mejorar el comportamiento de los trabajadores, las condiciones y el medio ambiente laboral (Secretaría General de la Alcaldía Mayor de Bogotá, 2015). "Además de mejorar los resultados en la prevención de los incidentes, accidentes y enfermedades en el lugar de trabajo por medio de la gestión eficaz de los peligros y riesgos" (OIT, 2007).

1 La norma ISO 9000:2015 define Sistema de Gestión como "un conjunto de elementos de una organización interrelacionados o que interactúan para establecer políticas, objetivos y procesos para lograr estos objetivos".
La norma OHSAS 18001:2007 establece que el SGSST hace parte de la gestión de una organización para desarrollar e implementar su política de SST y gestionar sus riesgos de SST, esto confirma que el SG-SST debería incorporarse e integrarse en la gestión estratégica de las empresas (Molano y Arévalo, 2013), "integrar la política y las acciones de Seguridad y Salud en el Trabajo a la gestión de las organizaciones" (Rodríguez, 2012) para contribuir al cumplimiento de los objetivos estratégicos relacionados con el crecimiento, rentabilidad y sostenibilidad del negocio "a través de la estructuración de sistemas de trabajo seguros, saludables y económicamente viables" (Molano y Arévalo, 2013).

En cuanto a la participación, Nácher (2002) indica que "es un estado de cosas en el que se comparte algo que se habla-sabe-hace-tiene con otros, es un bien a demandar y, por tanto, a producir colectivamente, genera más beneficios netos que la inhibición" (p. 24). Las personas hacen posible la participación, de manera directa 0 indirecta se involucran en asuntos de interés individual y colectivo a través de diferentes formas o mecanismos de participación para comunicarse, compartir ideas e inquietudes, opinar, analizar y tomar decisiones en relación a los temas que les afectan 0 benefician.

En la figura 1 se presentan las principales características de la participación.

Son los individuos quienes permiten el progreso de la organización y llevar a buen término los resultados esperados. Gaviria cita en su tesis de grado a Catillo e indica que "los problemas de las organizaciones no los solucionan las máquinas, la tecnología y demás recursos económicos por sí solos, sino que es la energía humana con sus conocimientos, experiencia y creatividad, canalizados a través de una dirección más participativa la que puede jalonarlas hacia niveles jerárquicos superiores de productividad y competitividad (Gaviria, 2010). 
Figura 1. Características de la participación.

\begin{tabular}{|c|c|c|c|c|}
\hline $\begin{array}{c}\text { Está influenciada } \\
\text { por: }\end{array}$ & $\begin{array}{l}\text { Requiere de los } \\
\text { individuos: }\end{array}$ & Se comparten: & A través de: & Con el fin de: \\
\hline $\begin{array}{l}\text { - Intereses } \\
\text { comunes } \\
\text { - Beneficios } \\
\text { - Necesidades } \\
\text { - Asuntos que } \\
\text { afectan a las } \\
\text { personas } \\
\text { - Búsqueda de } \\
\text { soluciones }\end{array}$ & $\begin{array}{l}\text {-Aprender } \\
\text { - Involucrarse } \\
\text { - Comunicar } \\
\text { - Implicarse } \\
\text { - Reportar } \\
\text { - Compartir } \\
\text { - Opinar } \\
\text { - Discutir } \\
\text { - Cooperar } \\
\text { - Dar y recibir } \\
\text { - Influir } \\
\text { - Tomar decisiones }\end{array}$ & $\begin{array}{l}\text {-Ideas } \\
\text { - Opiniones } \\
\text { - Inquietudes } \\
\text { - Información } \\
\text { - Experiencias } \\
\text { - Intuiciones }\end{array}$ & $\begin{array}{l}\text { - Mecanismos de } \\
\text { participación } \\
\text { - Representantes } \\
\text { intercambio de } \\
\text { información } \\
\text { - Consulta } \\
\text { - Codecisión }\end{array}$ & $\begin{array}{l}\text { - Generar nuevas } \\
\text { ideas para dar } \\
\text { soluciones y mejoras. } \\
\text { - Favorecer el } \\
\text { desarrollo y } \\
\text { crecimiento personal } \\
\text { e institucional. } \\
\text { - Potenciar las } \\
\text { acciones. El aporte } \\
\text { de varios individuos } \\
\text { es mayor que el de } \\
\text { uno solo. } \\
\text { - Promover la } \\
\text { democracia. } \\
\text { - Hacer efectivos } \\
\text { otros derechos }\end{array}$ \\
\hline
\end{tabular}

Fuente: elaboración propia.

Ahora bien, la participación constituye un derecho y un deber para las personas. La Declaración Universal de los Derechos Humanos (1948) define que: "toda persona tiene derecho a tomar parte libremente en la vida cultural de la comunidad, a gozar de las artes y a participar en el progreso científico y en los beneficios que de él resulten", así mismo, "toda persona tiene derecho a participar en el gobierno de su país, directamente 0 por medio de representantes libremente escogidos".

Para lograr la participación del personal, es necesario el liderazgo y firme compromiso por parte de los directivos de la organización para el funcionamiento eficaz y eficiente del Sistema de Gestión de Seguridad y Salud en el Trabajo (OTI, 2011). A saber, los jefes de área son los representantes del empleador de acuerdo con lo establecido en el Código Sustantivo de Trabajo colombiano, deben asumir una responsabilidad integral y activa con el SG-SST, ser ejemplo a seguir por parte de sus trabajadores, participar en la gestión de los riesgos de seguridad y salud en el trabajo generados por los procesos o actividades de su área y promover la participación de su equipo. A su vez, "los trabajadores deben participar activamente como beneficiarios y actores esenciales del SG-SST" (OIT, 2011).

La gestión de la Seguridad y Salud en el Trabajo requiere de la participación de cada uno de los individuos de la empresa, es decir que el presidente 0 gerente, los supervisores, jefes, personas con personal bajo su responsabilidad y los trabajadores deben participar, por consiguiente contribuir a la gestión de los riesgos de la organización y ser parte activa para el mejoramiento del SG-SST. La participación, según Rodríguez (2012), es el "sistema mediante el cual los empleados de una empresa son asociados a sus beneficios y eventualmente a su gestión”. 
Figura 2. Mecanismos de participación.

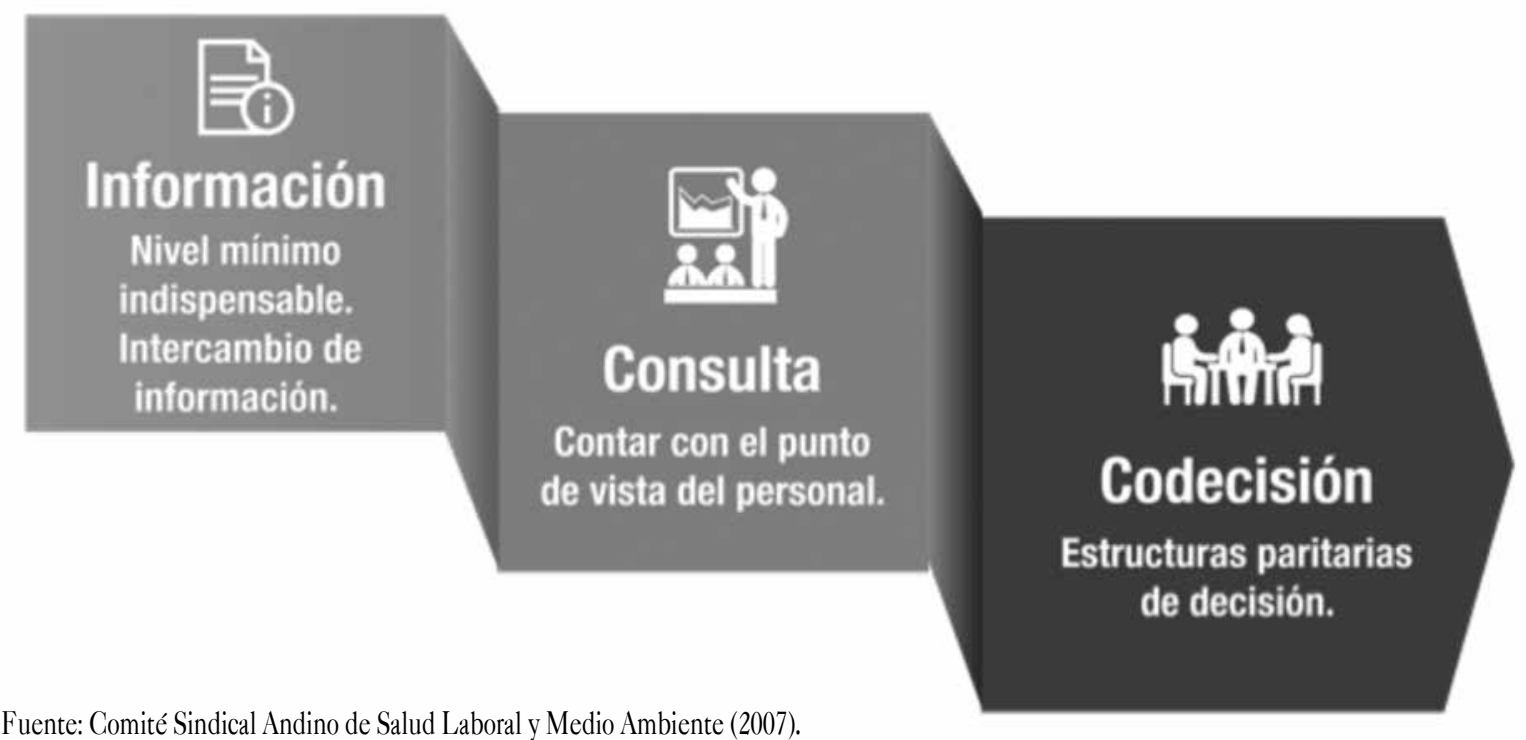

"El Instituto Laboral Andino describe tres mecanismos de participación: el intercambio de información, la consulta y la codecisión; iniciando desde una participación distante hasta una participación democrática que permite la generación de nuevas ideas en beneficio de todos" (Comité Sindical Andino de Salud Laboral y Medio Ambiente, 2007, p. 14). El intercambio de información es necesario para que se presenten los mecanismos de participación de consulta y codecisión. Gaviria menciona que la participación es imposible sin información, los empleados no pueden entender cómo funciona el proceso, si no recibe información, ni pueden mejorarlo mediante sus contribuciones. "Sin los conocimientos y la falta de formación la organización se enfrenta a un claro obstáculo para la participación. Solo pueden funcionar con empleados competentes, dotados de habilidades para realizar tareas con eficacia" (Gaviria, 2010).

Respecto al intercambio de información, la norma ISO 9001 definió algunos elementos que deben contener las comunicaciones internas en la organización, como son qué comunicar, cuándo comunicar, a quién comunicar, cómo comunicar, quién comunica (Norma
Técnica Colombiana, NTC-ISO 9001:2015). Estos requisitos son fundamentales para cualquier tipo de comunicación; ello implica realizar una planeación de las comunicaciones internas para lograr que todos los colaboradores reciban información suficiente, de calidad y oportunamente. A través de la consulta se escucha al trabajador, sus opiniones, sugerencias, inconformidades e ideas; la empresa cuenta con el punto de vista de su personal.

El mecanismo de participación ideal corresponde a la codecisión, la cual permite a los individuos no solamente compartir sus intereses, sino además ser parte de las decisiones colectivas que se tomen y ser conscientes de participar voluntariamente. Gaviria (2010) menciona que para que la participación obtenga resultados positivos deseados, es necesario que los individuos tengan la capacidad y el deseo de participar. Los trabajadores no deberán limitar su participación a una cooperación pasiva y a respetar las normas de seguridad. Para alcanzar el nivel de protección más eficaz de su propia seguridad y salud, los trabajadores necesitan aprovechar plenamente los procedimientos de participación en su lugar de trabajo (Gaviria, 2010). 
La participación aumenta cuando el personal tiene claro ciertos aspectos como sus responsabilidades, funciones, mecanismos de participación y sabe cómo contribuye a la organización. Parte de esto se puede lograr a través de la formación del personal y de acuerdo con Gaviria (2010), las actividades que promuevan la formación entre los colaboradores conducen a la participación. Esta formación debe ser constante y sostenible en todos los niveles de la organización.

Igualmente, se requiere de liderazgo; el liderazgo debe presentarse desde diferentes figuras en la empresa, que incluye a todos los niveles de la organización, incluso a los responsables del SG-SST. Actualmente las organizaciones se enfrentan a diferentes retos a nivel organizacional, de mercados, clientes, competencia, tecnología, entre otros factores a tener en cuenta, Gil e Ibarra (2007) mencionan que un líder debe resolver problemas complejos y debe tener las competencias necesarias para resolver situaciones vagamente estructuradas con alto nivel de efectividad; también indican que:

El líder debe olvidarse de la visión tradicional y debe ser visto como un agente de cambio con aptitudes, actitudes, conocimientos, con habilidades personales, capacidad de dirección que permita cambiar en forma deliberada o espontánea la dinámica del equilibrio inmovilizador en las organizaciones (Gil e Ibarra, 2007).

Los líderes del Sistema de Gestión de Seguridad y Salud en el Trabajo y el Comité Paritario de Seguridad y Salud en el Trabajo - COPASST, deben orientar la planeación, organización, implementación, mantenimiento, evaluación y mejoramiento del SG-SST; deben llevar el timón en la organización para lograr la prevención de los riesgos laborales. Sin duda alguna, su función debe contar con el respaldo del nivel estratégico de la organización y tener la capacidad de involucrar paulatinamente a todo el personal.

\section{METODOLOGÍA}

Las fases seguidas para el desarrollo de la investigación se presentan en la figura 3 y la tabla 1 a continuación.

Figura 3. Fases de la investigación.
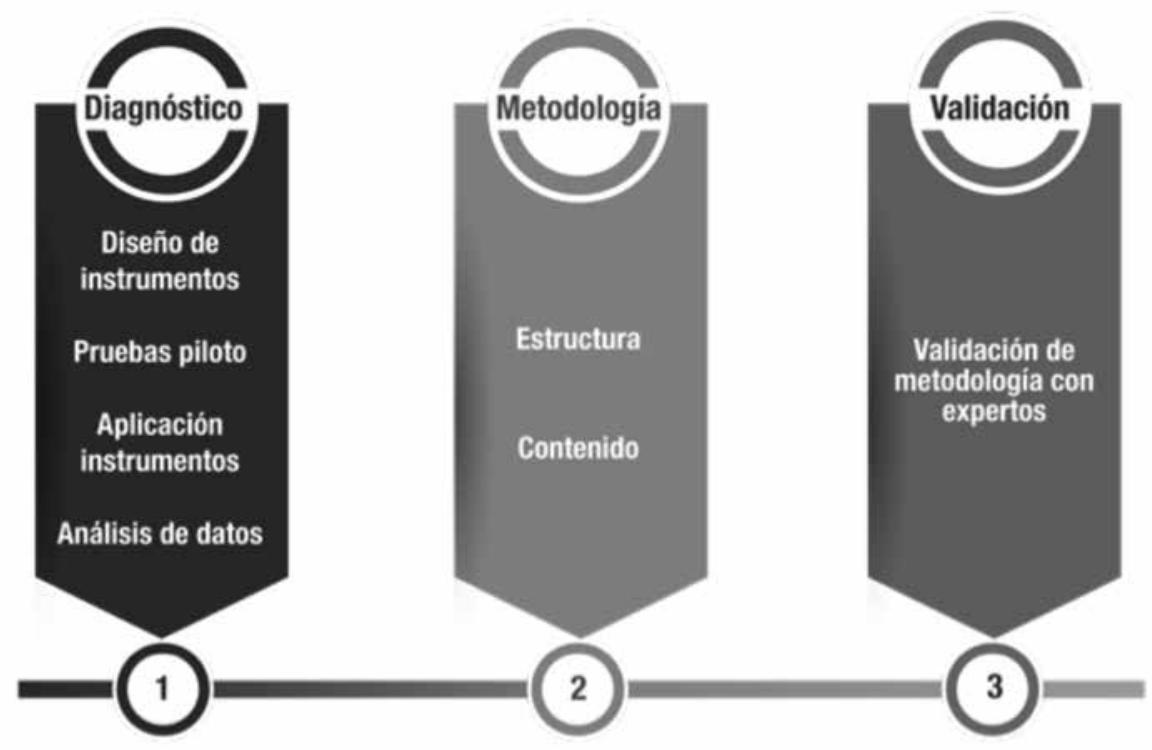

Fuente: elaboración propia. 
Tabla 1. Descripción de las fases de la investigación.

\begin{tabular}{|c|c|c|}
\hline N. & Fases & Descripción \\
\hline 1 & Diagnóstico & $\begin{array}{l}\text { En esta primera fase se realizaron actividades relacionadas con la evaluación e identificación de } \\
\text { los mecanismos de la participación del personal en el SG-SST: } \\
\text { Diseño de encuesta } \\
\text { Diseño de entrevista } \\
\text { Revisión y validación de encuesta por expertos } \\
\text { Pruebas pilotos de la encuesta } \\
\text { Aplicación de los instrumentos } \\
\text { Procesamiento de datos } \\
\text { Análisis de datos y resultados }\end{array}$ \\
\hline 2 & $\begin{array}{l}\text { Definición de metodología } \\
\text { de participación }\end{array}$ & $\begin{array}{l}\text { La segunda fase comprendió el diseño de la metodología para fortalecer la participación del } \\
\text { personal en el SG-SST, según análisis de los resultados de los instrumentos y marco referencial. }\end{array}$ \\
\hline 3 & $\begin{array}{l}\text { Validación de la metodología } \\
\text { de participación }\end{array}$ & En esta fase se realizó la validación de la metodología propuesta a través de juicio de expertos. \\
\hline
\end{tabular}

Fuente: elaboración propia.

Se diseñó una encuesta para evaluar la participación del personal de los niveles táctico y operativo en el SG-SST e identificar sus mecanismos de su participación. Se incluyeron preguntas sobre datos básicos del encuestado como área de trabajo, cargo, edad, estudios, preguntas para evaluar cada uno de los mecanismos de participación de información, consulta y codecisión; así como preguntas sobre frecuencia de la participación y relacionadas con factores que motivan y evitan la participación. También se incluyeron preguntas enfocadas únicamente para los miembros del COPASST y responsables del SG-SST.

La encuesta fue sometida a validación por 9 expertos, de los cuales 7 atendieron la solicitud. 5 de ellos con estudios y experiencia en Seguridad y Salud en el Trabajo, 1 profesional en Antropología y 1 profesional en Comunicación Social y Periodismo. Se recibieron 52 observaciones por parte de los 7 expertos, de las cuales 40 , equivalentes al $76.9 \%$, fueron aplicadas a la encuesta para mejorar su contenido, y 12 observaciones fueron consideradas como no aplicables. Para el personal del nivel estratégico, se diseñó una entrevista semiestructurada de 16 preguntas sobre información, consulta y codecisión. La entrevista no fue sometida a validación por los expertos.

Con base en el marco referencial, la experiencia del investigador y teniendo en cuenta que a través de la encuesta se logró cuantificar el porcentaje de participación del personal en el SG-SST, se definió una escala de valoración, a través de 5 niveles, incluyendo la interpretación cuantitativa y cualitativa. Esta escala también fue sometida a validación por los 7 expertos que validaron la encuesta, de los cuales 5 contestaron afirmativamente.

Posterior a los ajustes realizados a la encuesta y a la escala de valoración del nivel de participación, se realizó una prueba piloto, a través de la plataforma en línea de Google Formularios en la que participaron 15 colaboradores de una empresa de transporte terrestre de hidrocarburos y sus derivados. Se identificó que el nivel de participación del personal en el SG-SST fue 
Tabla 2. Interpretación del nivel de participación en el SG-SST.

\begin{tabular}{|l|l|}
\hline \multicolumn{1}{|c|}{ Nivel de participación } & \multicolumn{1}{c|}{ Descripción } \\
\hline I Incipiente & $\begin{array}{l}\text { Participación mínima en el SG-SST; el personal está iniciándose a participar; resultados en la encuesta inferiores } \\
\text { al } 30 \% .\end{array}$ \\
\hline II Baja & $\begin{array}{l}\text { Participación baja en el SG-SST; el personal participa de forma obligatoria; por lo general el mecanismo de } \\
\text { participación mejor desarrollado es el de información; resultados en la encuesta entre el 30\% al 50\%. }\end{array}$ \\
\hline III Significativa & $\begin{array}{l}\text { Participación media en el SG-SST; el personal participa en las actividades básicas o programadas; mecanismos de } \\
\text { participación medianamente desarrollados; resultados en la encuesta entre el 51 \% y el 70 \%. }\end{array}$ \\
\hline IV Elevada & $\begin{array}{l}\text { Participación alta en el SG-SST; el personal participa en las actividades voluntariamente; mecanismos de } \\
\text { participación de información y consulta desarrollados; resultados en la encuesta entre el 71\% al 90\%. }\end{array}$ \\
\hline V Plena & $\begin{array}{l}\text { Participación ideal y máxima en el SG-SST; el personal participa voluntariamente, está comprometido y es } \\
\text { consciente de su rol con el SG-SST; los mecanismos de participación de información, consulta y codecisión son } \\
\text { maduros; resultados en la encuesta superiores al 90 \%. }\end{array}$ \\
\hline
\end{tabular}

Fuente: elaboración propia.

de $56.8 \%$, equivalente a un nivel de participación significativa; el mecanismo de participación de información obtuvo un $61.8 \%$ (significativa), consulta $34.4 \%$ (baja), codecisión $54.9 \%$ (significativa), COPASST y líderes del SG-SST 76.2\% (elevada).

Con la aplicación de la prueba piloto, se evidenció que el instrumento logró recolectar los datos esperados; se identificó el nivel de participación del personal e identificaron sus mecanismos de participación. La prueba piloto permitió identificar algunas mejoras en cuanto al diseño de la encuesta en la plataforma en línea y la agrupación de los resultados para el análisis una vez descargada la base de datos. La escala de interpretación del nivel de participación también fue ajustada.

A partir de una población total de 909 colaboradores de la empresa objeto de estudio, se realizó un muestreo estratificado con afijación proporcional; la población se dividió en subgrupos o unidades de negocio según la estructura organizacional; de cada subgrupo se seleccionó una muestra proporcional a su tamaño, entre 44 \% a 50 \%. La recolección de los datos se realizó a través de la herramienta web SurveyMonkey para el personal de los niveles operativo y táctico y por medio de entrevistas para el personal del nivel estratégico. 


\section{RESULTADOS Y DISCUSIÓN}

Tabla 3. Datos demográficos del personal encuestado.

\begin{tabular}{|c|c|c|}
\hline Descripción & Participantes & Porcentaje \\
\hline \multicolumn{3}{|l|}{ Distribución por género } \\
\hline Femenino & 193 & $60.30 \%$ \\
\hline Masculino & 127 & $39.70 \%$ \\
\hline \multicolumn{3}{|l|}{ Distribución por edad (años) } \\
\hline 18 a 30 & 133 & $41.60 \%$ \\
\hline 31 a 40 & 124 & $38.80 \%$ \\
\hline 41 a 50 & 53 & $16.60 \%$ \\
\hline Mayor a 50 & 10 & $3.10 \%$ \\
\hline \multicolumn{3}{|c|}{ Distribución por nivel de gestión } \\
\hline Nivel táctico & 173 & $54.10 \%$ \\
\hline Nivel operativo & 147 & $45.90 \%$ \\
\hline \multicolumn{3}{|c|}{ Distribución por nivel académico } \\
\hline Técnico & 123 & $38.40 \%$ \\
\hline Profesional & 94 & $29.40 \%$ \\
\hline Tecnólogo & 59 & $18.40 \%$ \\
\hline Especialista & 24 & $7.50 \%$ \\
\hline Bachiller & 17 & $5.30 \%$ \\
\hline Magíster & 3 & $0.90 \%$ \\
\hline \multicolumn{3}{|c|}{ Distribución por antigüedad en la empresa } \\
\hline Más de 5 años & 186 & $58.10 \%$ \\
\hline De 2 a 5 años & 62 & $19.40 \%$ \\
\hline 1 a 2 años & 32 & $10.00 \%$ \\
\hline De 3 meses a 1 año & 32 & $10.00 \%$ \\
\hline Menos de 3 meses & 8 & $2.50 \%$ \\
\hline
\end{tabular}

Fuente: elaboración propia.

193 mujeres y 127 hombres completaron la encuesta; contó con la participación de 133 colaboradores entre 18 y 40 años, 124 de 31 a 40 años, 53 de 41 a 50 años y 10 mayores de 50 años. 173 colaboradores del nivel táctico de la organización, quienes tienen una o más personas a su cargo completaron la encuesta, mientras que del personal operativo participaron 147 colaboradores. Principalmente, los participantes de la muestra fueron colaboradores con un nivel académico técnico (123), seguido por los profesionales (94) y tecnólogos (59); en menor proporción personal con especialización, bachillerato y maestría. 186 colaboradores de la 
Fiǵura 4. Nivel de participación en el SG-SST.

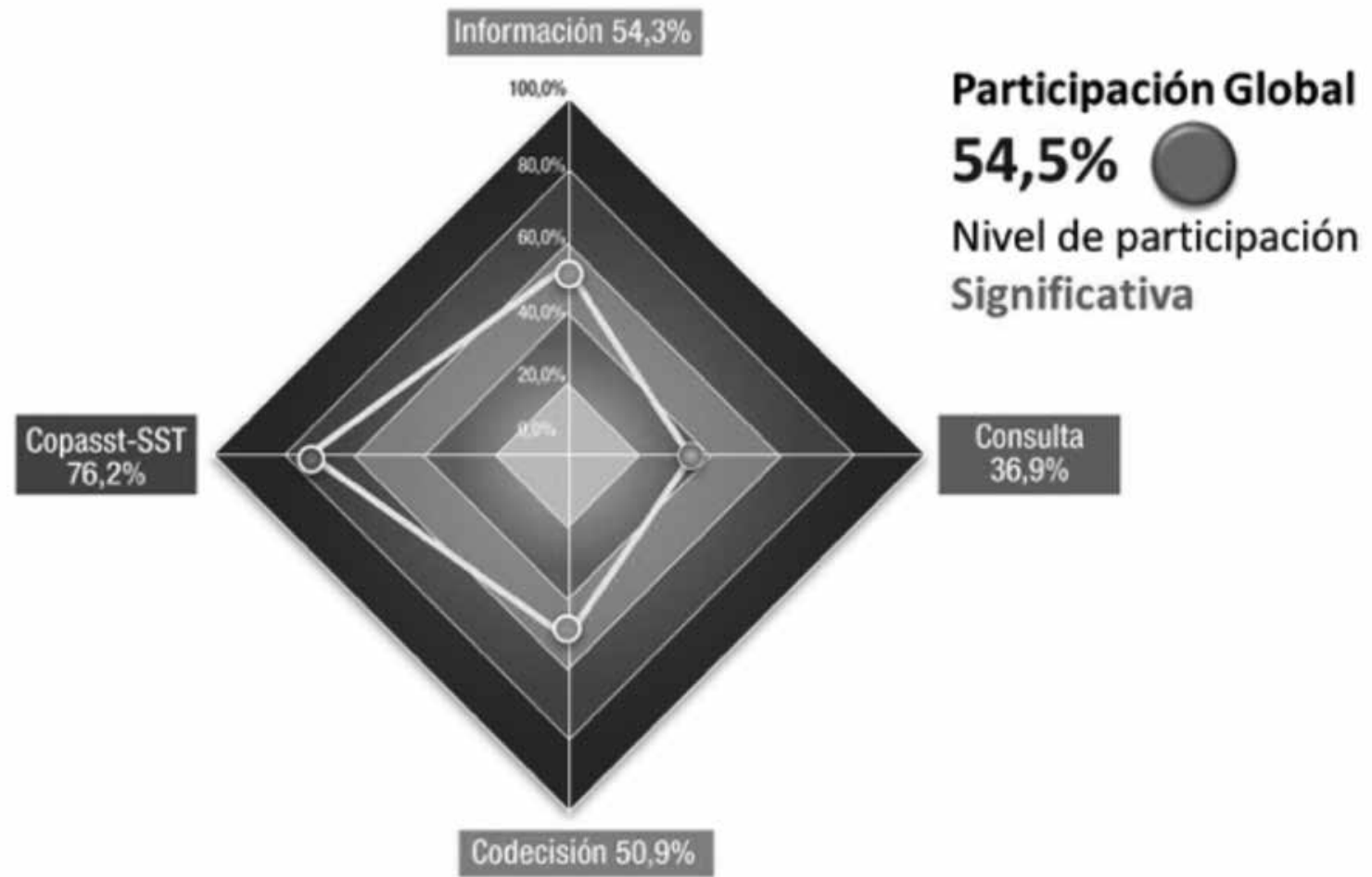

Fuente: elaboración propia.

muestra contaban con una antigüedad en la empresa mayor a 5 años, seguido por colaboradores con antigüedad menor a 2 años y de 2 a 5 años.

Servicios Aeroportuarios, Operaciones Técnicas y Comercial fueron las áreas con mayor número de encuestados. Estas áreas cuentan con la mayor cantidad de trabajadores. En las ciudades principales como Bogotá, Medellín y Cali hay mayor cantidad de colaboradores; el número de encuestados por ciudad fue proporcional al número de colaboradores.

Para hallar el porcentaje de participación se agrupó la proporción de respuestas positivas y respuestas negativas por cada una de las preguntas. La participación a través de los mecanismos de información y codecisión obtuvo resultados de $54.3 \%$ y $50.9 \%$ respectivamente, equivalentes a un nivel de participación significativa; el mecanismo de participación de consulta obtuvo un resultado de $36.9 \%$ equivalente a un nivel de participación baja.

A través de un promedio ponderado, la empresa obtuvo un resultado de $54.5 \%$, equivalente a un nivel de participación significativa, es decir que el nivel de participación del personal operativo y táctico se encontró en un nivel intermedio, por encima de la participación incipiente y baja, con oportunidades de mejora y fortalecimiento para lograr un nivel elevado o pleno, superior al $70 \%$. El nivel de participación del COPASST y de los líderes de SST fue elevada (76.2\%); el personal del nivel táctico obtuvo un resultado de $48.3 \%$ y el personal operativo $47.7 \%$, equivalentes a un nivel de participación baja. 
El personal con antigüedad entre 1 y 2 años obtuvo el mejor resultado (50.2\%), el menor porcentaje fue el personal con antigüedad menor a 3 meses (35.4\%); el personal bachiller obtuvo el mejor resultado con $58.1 \%$, seguido por los especialistas y en último lugar el personal con maestría (38.8\%); los colaboradores mayores de 50 años obtuvieron el mejor resultado (59\%) y el personal con menor resultado fueron los de 31 a 40 (46.6\%).

Figura 5. Nivel de participación por áreas operativas.

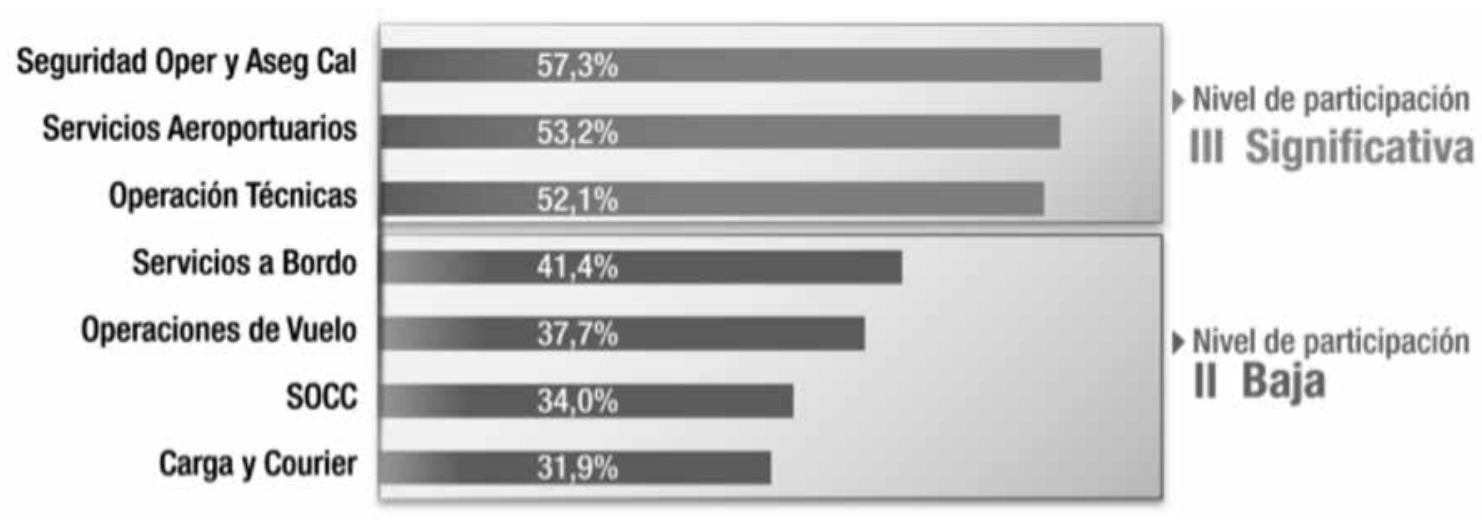

Fuente: elaboración propia.

Figura 6. Nivel de participación por áreas administrativas.

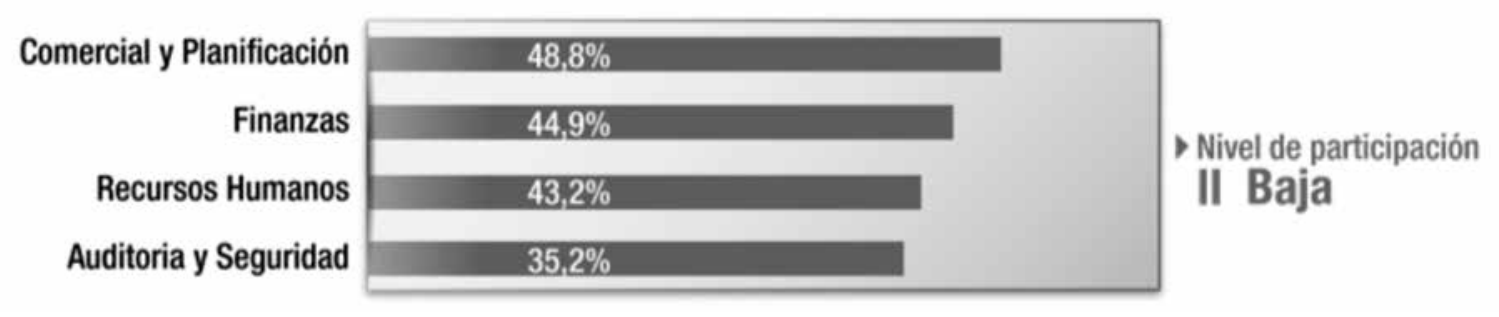

Fuente: elaboración propia.

Las áreas de Seguridad Operacional y Aseguramiento de la Calidad, Servicios Aeroportuarios y Operaciones Técnicas obtuvieron resultados superiores al $50 \%$, equivalentes a un nivel de participación significativa; las áreas de Comercial, Finanzas, Recursos Humanos y
Servicio a Bordo, obtuvieron un nivel de participación baja (entre el $40 \%$ y $50 \%$ ); las áreas de Operaciones de Vuelo, Auditoría y Seguridad, SOCC y Carga y Courrier obtuvieron resultados por debajo del $40 \%$, también equivalente a un nivel de participación baja. 
Tabla 4. Mecanismos de participación del personal en el SG-SST.

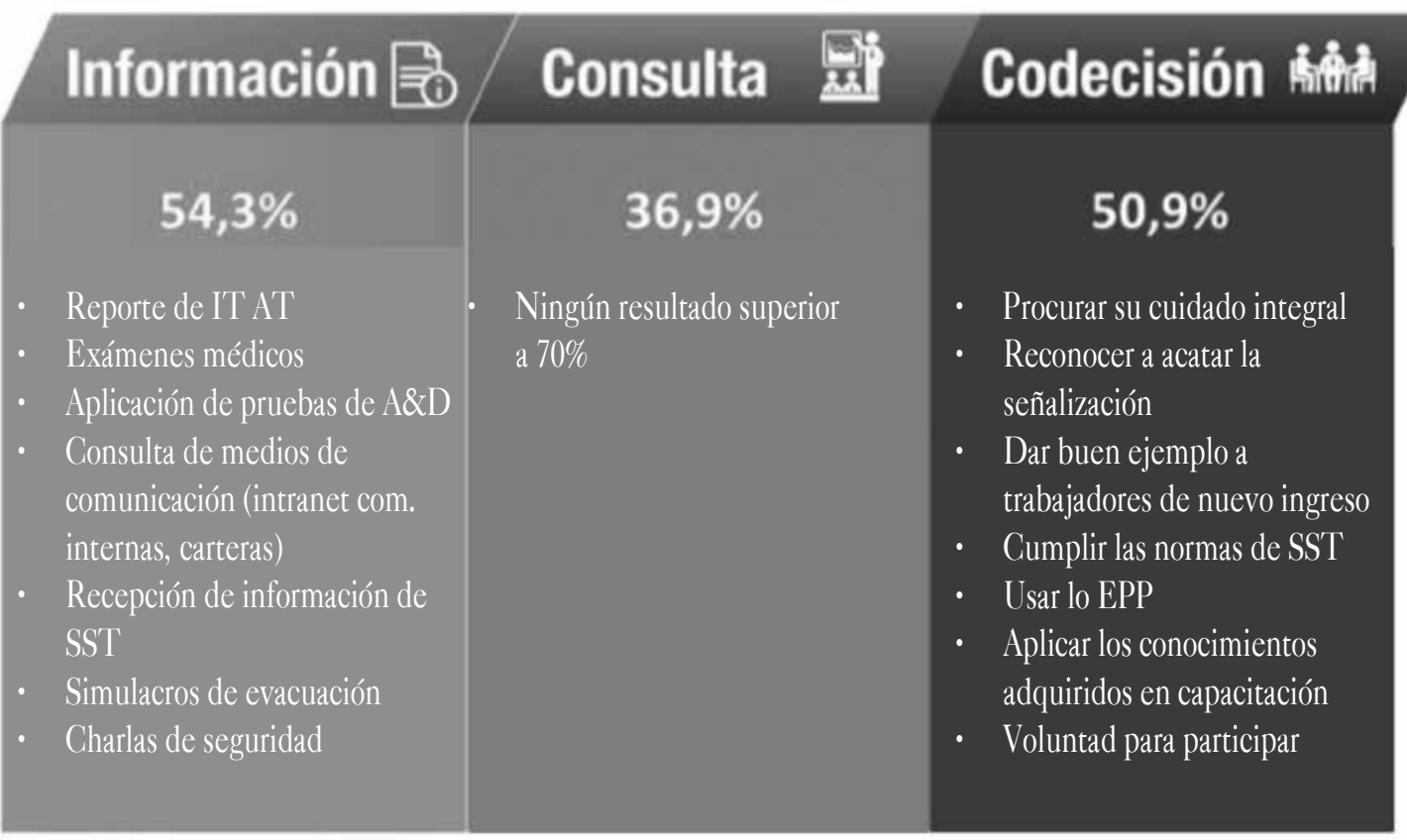

Fuente: elaboración propia.

Entre el nivel táctico y el nivel operativo, el mecanismo de participación que mayor se presentó fue el de información (54.3\%), seguido por codecisión (50.9\%) y en tercer lugar la consulta $(36.9 \%)$; tanto en el nivel táctico como en el nivel operativo, el mecanismo de participación más representativo fue el de información, seguido por codecisión y consulta; mismo orden que a nivel compañía. Por su parte, el COPASST y líderes SST obtuvieron el mayor resultado en el mecanismo de consulta (79.2\%), seguido por codecisión $(70.3 \%)$ e información (69\%).

Tabla 5. Nivel de participación del personal directivo en el SG-SST.

\begin{tabular}{|c|c|c|}
\hline Cargo & Nivel de participación & Descripción \\
\hline Vicepresidente operaciones & Elevada & $\begin{array}{l}\text { Durante la entrevista se evidenció que el vicepresidente de operaciones } \\
\text { es quien mayor participación tiene en el SG-SST. Su participación es alta; } \\
\text { participa en las actividades voluntariamente; se evidenció que los mecanismos } \\
\text { de participación de consulta y codecisión se encuentran desarrollados. }\end{array}$ \\
\hline Presidente & Significativa & \multirow{3}{*}{$\begin{array}{l}\text { Se evidenció durante las entrevistas que el presidente, vicepresidente de } \\
\text { finanzas y el director técnico tienen una participación media en el SG-SST; } \\
\text { el personal participa en las actividades básicas o programadas; mecanismos de } \\
\text { participación medianamente desarrollados. }\end{array}$} \\
\hline Vicepresidente finanzas & Siginificativa & \\
\hline Director técnico & Significativa & \\
\hline Director operaciones de vuelo & Baja & \multirow{3}{*}{$\begin{array}{l}\text { Se evidenció en las entrevistas que los directores de auditoría y seguridad, } \\
\text { operaciones de vuelo y mercadeo tienen una participación baja en el } \\
\text { SG-SST; el personal participa de forma obligatoria o cuando son invitados; } \\
\text { los mecanismos de participación de información y consulta están poco } \\
\text { desarrollados. }\end{array}$} \\
\hline Director auditoría y seguridad & Baja & \\
\hline Director de mercadeo & Baja & \\
\hline
\end{tabular}

Fuente: elaboración propia. 
Tabla 6. Mecanismos de participación del personal directivo en el SG-SST.

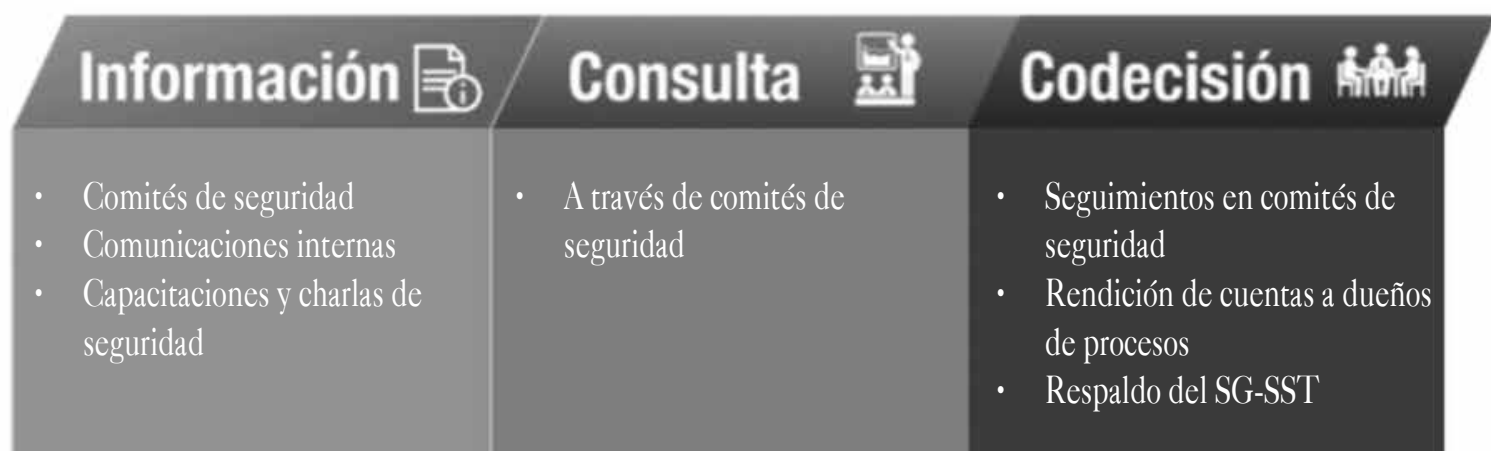

Fuente: elaboración propia.

Los mecanismos de participación del personal del nivel estratégico están directamente relacionados con los comités de seguridad; a través de estos espacios, el presidente, vicepresidentes y directores de la empresa se enteran de los principales indicadores y actividades del SG-SST. En los comités de seguridad se analiza mensualmente los índices de accidentalidad, de ausentismo, investigación de accidentes de trabajo y los planes de acción resultantes de las investigaciones; también es el espacio donde el personal del nivel táctico rinde cuentas sobre su gestión en SST. Los directivos también informaron que participan a través de la asistencia a capacitaciones o charlas de seguridad.

Tabla 7. Participación del personal en el SG-SST.

\begin{tabular}{|c|c|c|}
\hline Nivel de gestión & Área/Cargo & Nivel de participación \\
\hline \multirow{11}{*}{ Operativo y táctico } & Seguridad oper y aseg cal & Significativa \\
\hline & Servicios aeroportuarios & Significativa \\
\hline & Operaciones técnicas & Significativa \\
\hline & Servicios a bordo & Baja \\
\hline & Operaciones de vuelo & Baja \\
\hline & SOCG & Baja \\
\hline & Carga y courier & Baja \\
\hline & Comercial y planificación & Baja \\
\hline & Finanzas & Baja \\
\hline & Recursos humanos & Baja \\
\hline & Auditoría y seguridad & Baja \\
\hline \multirow{8}{*}{ Estratégico } & Vicepresidente de operaciones & Elevada \\
\hline & COPASST y SST & Elevada \\
\hline & Presidente & Significativa \\
\hline & Vicepresidente finanzas & Significativa \\
\hline & Director técnico & Significativa \\
\hline & Director operaciones de vuelo & Baja \\
\hline & Director auditoría y seguridad & Baja \\
\hline & Director de mercadeo & Baja \\
\hline
\end{tabular}

Fuente: elaboración propia. 
La participación del personal del nivel estratégico en el SG-SST estuvo entre baja, significativa y elevada; 3 directivos obtuvieron un nivel de participación baja, otros 3 significativa y 1 elevada; en el nivel de gestión estratégica se incluyó el COPASST y los líderes de SST. Tomando como referencia los 3 directivos con nivel de participación significativa, 1 directivo, el COPASST y los líderes SST con participación elevada, se puede afirmar que el personal del nivel estratégico obtuvo un nivel de participación significativa.

Por lo anterior, se evidenció que el nivel de participación del personal en el SG-SST fue significativa. En cuanto a los mecanismos de participación, se identificó que en los niveles táctico y operativo, el mecanismo de participación más representativo fue el de información, seguido por codecisión y consulta; se identificó que el mecanismo de participación del nivel estratégico más desarrollado es el de información, a través de comités de seguridad, comunicaciones internas, capacitaciones y charlas de seguridad.

\section{Metodología propuesta para fortalecer la participación}

A partir de los resultados de la evaluación de la participación, además de las referencias descritas en el marco teórico, legal y normativo, se diseñó una metodología para fortalecer la participación del personal en el SG-SST.

La metodología describe los principios para el fortalecimiento de la participación del personal como son: el compromiso, la claridad, la comunicación, la creatividad, la consistencia, la credibilidad y la continuidad.

El punto de partida para su aplicación es la elaboración del contexto y diagnóstico inicial de la organización. A través de instrumentos como encuestas y entrevistas, además del análisis de los procesos, la estructura organizacional, estadísticas de accidentes de trabajo y
Figura 7. Principios para el fortalecimiento de la participación en el SG-SST.

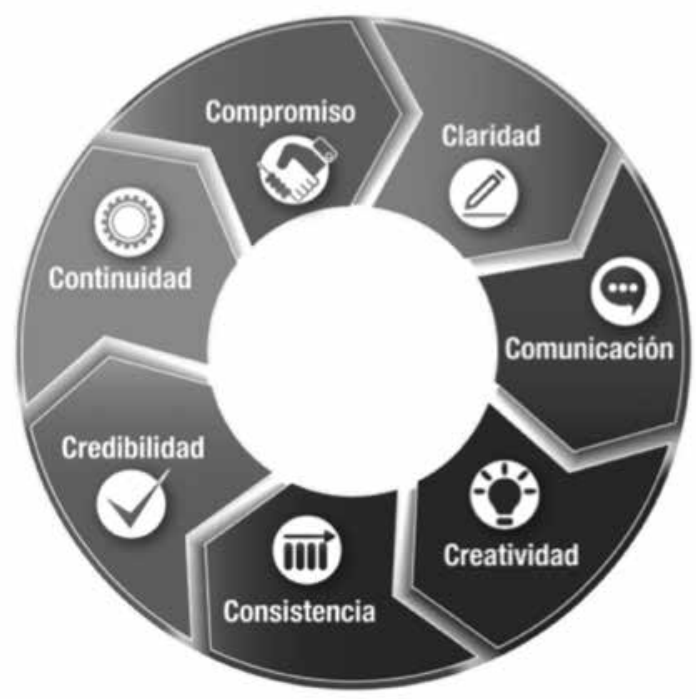

Fuente: elaboración propia.

enfermedades laborales, se obtiene información para identificar las fortalezas, debilidades, oportunidades y amenazas del SG-SST. La metodología describe cómo elaborar la política, objetivos y planificación del SGSST, con énfasis en la participación; también describe la información documentada requerida.

La metodología tiene como base el ciclo de mejoramiento continuo PHVA (planear, hacer, verificar, actuar) y los 3 mecanismos de participación: información, consulta y codecisión; se describe lo que la organización debería aplicar en cada mecanismo.

5 expertos con especialización o maestría en Seguridad y Salud en el Trabajo contestaron 9 preguntas para la validación de la metodología propuesta. En promedio, los expertos respondieron positivamente el $87 \%$ de las preguntas, indicando que la metodología cumplía los criterios de validación definidos.

El $100 \%$ de los expertos indicaron que las etapas de la metodología estaban descritas de manera ordenada, 
Figura 8. Proceso para el fortalecimiento de la participación en el SG-SST.

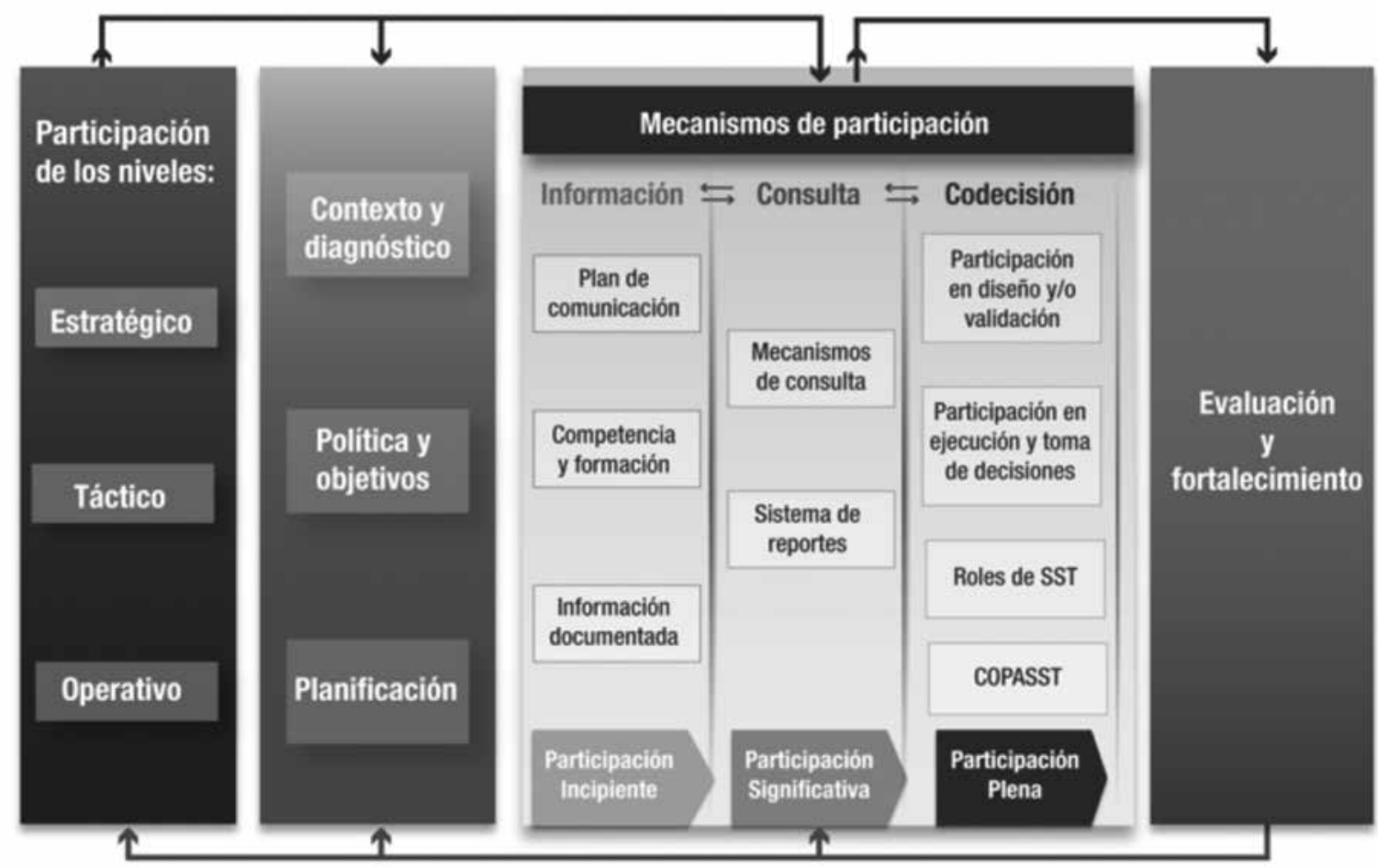

Fuente: elaboración propia.

clara y lógica; que la metodología era factible, su contenido era de calidad y su aplicación tendría impacto positivo en el desempeño eficaz del SG-SST de la empresa que la aplique; el $83.3 \%$ de los expertos indicaron que el diseño de la metodología, su presentación y contenido era fácilmente entendible y cumplía con el objetivo para el cual fue diseñada.

El $66.7 \%$ de los expertos indicaron que el contenido de la metodología fue suficiente y pertinente para fortalecer la participación, que las acciones descritas en cada mecanismo de participación (información, consulta y codecisión) fueron claras y suficientes para fortalecer la participación del personal en el SG-SST.

Los expertos realizaron algunas observaciones para el mejoramiento de la metodología: la inclusión del principio de liderazgo como enfoque de empoderamiento de todos los niveles de la organización, la toma de conciencia por parte de los trabajadores y los valores corporativos que representan las convicciones del personal y la identidad de la organización; revisión general de la metodología en cuanto a cómo lograr el fortalecimiento, así como la revisión de la redacción. Uno de los expertos indicó revisar el concepto de metodología y ajuste en la propuesta, teniendo en cuenta que consideró que el documento enviado correspondía a una serie de lineamientos y no a una metodología propiamente dicha.

Se revisó la metodología y se hicieron algunos ajustes en cuanto a forma y contenido; se incluyó un instrumento para la evaluación del desempeño y avance del SG-SST, además de algunos documentos sobre la aplicación de la metodología en la empresa: la política de Seguridad y Salud en el Trabajo y el reglamento de higiene y seguridad industrial, objetivos y cronograma del SG-SST y un video sobre su lanzamiento. 
Metodología para fortalecer la participación del personal en el Sistema de Gestión de Seguridad y Salud en el Trabajo en una empresa del sector aeronáutico

\section{CONCLUSIONES}

Se diseñó la metodología para fortalecer la participación del personal de los niveles estratégico, táctico y operativo de la empresa en el SG-SST, siguiendo el ciclo de mejoramiento continuo PHVA (planear, hacer, verificar, actuar) y los mecanismos de participación información, consulta y codecisión. 5 expertos con especialización 0 maestría en Seguridad y Salud en el Trabajo participaron en su validación y respondieron positivamente el 87 $\%$ de las preguntas, indicando que la metodología cumplió con los criterios de validación definidos. Conforme a su estructura y contenido, la metodología puede ser aplicada por cualquier organización que tenga como propósito fortalecer la participación del personal de los niveles estratégico, táctico y operativo en el SG-SST.

Se evidenció que el nivel de participación del personal de los niveles estratégico, táctico y operativo en el SG-SST fue significativa, a diferencia de la primera hipótesis planteada, que indicaba que el nivel de participación del personal era baja; respecto a la segunda hipótesis se confirmó y se evidenció que el mecanismo de participación que se encuentra mejor desarrollado es el de "información". Los mecanismos de participación utilizados por parte del personal del nivel directivo son los comités de seguridad, comunicaciones internas y capacitación. El compromiso del personal directivo es esencial para el desempeño del SG-SST y se evidenció durante las entrevistas en cuanto al respaldo, seguimiento en comités y solicitud de rendición de cuentas al personal del nivel táctico.

Se confirmó que las áreas que tienen mejores resultados de participación del personal en el SG-SST son las áreas operativas de Seguridad Operacional y Aseguramiento de la Calidad, Servicios Aeroportuarios y Operaciones Técnicas; igualmente se confirmó que las áreas con menor participación son las áreas operativas como Operaciones de Vuelo, Carga, Despacho y Servicio a Bordo, así como las áreas administrativas.
Todos los directivos entrevistados estuvieron de acuerdo con las siguientes afirmaciones sobre el SG-SST: "Los trabajadores son los principales beneficiados del SG-SST en términos de bienestar, salud y calidad de vida para las personas" y "los resultados del SG-SST contribuyen al crecimiento, rentabilidad y sostenibilidad de la organización”.

El $90 \%$ de los colaboradores indicaron que la SST tiene por objeto mejorar las condiciones y el medio ambiente de trabajo en la empresa, prevenir accidentes de trabajo y enfermedades; el $82 \%$ reconocieron que la SST promueve el mantenimiento y mejoramiento de la salud física, mental y social de los trabajadores en sus actividades laborales. E1 $94 \%$ de los colaboradores consideraron que la participación de los trabajadores permite expresar opiniones y necesidades con el fin de lograr beneficios comunes y cumplir los objetivos de la empresa; y más del $90 \%$ informaron que consideran que el aporte y los resultados de la participación de varios individuos es mayor que el de uno solo en términos de SST y que la participación de todos los colaboradores en el Sistema de Gestión de SST trae efectos y resultados positivos para la organización.

\section{REFERENCIAS}

Agencia Europea para la Seguridad y la Salud en el Trabajo. (2012). Participación de los trabajadores en la seguridad y la salud en el trabajo. Una guía práctica. Recuperado de https://osha.europa.eu/ es/tools-and-publications/publications/reports/ workers-participation-in-OSH_guide

Betancourt, O. (2007). Enfoque alternativo de la salud y seguridad en el trabajo. IESS, Prevención es desarrollo 1, 3-16.

Comité Sindical Andino de Salud Laboral y Medio Ambiente. (2007). Los Comités de Saludy Seguridad 
en el Trabajo y la participación de las trabajadoras y los trabajadores. Instituto Laboral Andino.

Declaración Universal de Derechos Humanos. (1948). Resolución de la Asamblea General, aprobada el 10 de diciembre de 1948. Recuperado de https://www.derechos.net/doc/tratados/dudh.html

Dirección General de Riesgos Laborales y Comité Nacional de Salud Ocupacional del Ministerio de Trabajo. (2016). Plan Nacional de Salud Ocupacional 2013-2021. Bogotá. Recuperado de https:// www.oiss.org/IMG/pdf/PlanNacionalDeSeguridadySaludEnEITrabajo.pdf

Erazo, J. M. (2014). La Gestión de la Saludy Seguridad en el Trabajo y Promoción de la Salud y Seguridad en el Trabajo: revisión bibliográfica. Bogotá: Universidad Nacional de Colombia, sede Bogotá, programas de Salud Ocupacional.

Gaviria, N. R. (2010). La dirección participativa, un intento de reconstrucción del concepto y sus implicaciones para la organización. Maestría en Administración (MBA). Manizales: Universidad Nacional de Colombia.

Gil, I. M., e Ibarra, S. (2007). Incidencia del liderazgo en los factores críticos de éxito como estrategia competitiva empresarial. Revista Dimensión Empresarial, 12(2), 119.

Icontec. (2007). Sistemas de Gestión en Seguridad y Salud Ocupacional. Requisitos (NTC 18001:2007), 1. a edición. Bogotá.

Icontec. (2015). Norma Técnica Colombiana Norma ISO 9000:2015. Bogotá.

Icontec. (2015). Norma Técnica Colombiana NTC-ISO 9001:2015. Numeral 7.4. Bogotá.

Instituto Colombiano de Normas Técnicas y Certificación ICONTEG. (2007). NTG-OHSAS 18001,
Sistema de gestión en seguridad y salud ocupacional. Bogotá: el Instituto.

Instituto Laboral Andino. (2007). Los comités de la Salud y Seguridad en el Trabajo y la participación de las trabajadoras y trabajadores, 1. a edición. Lima, Perú.

Mendoza, Ó., y Sánchez, M. (Enero de 2012). Gestión Integral de Riesgos, 4. edición. Bogotá: Consorcio Gráfico Ltda.

Ministerio de Trabajo. (2013). Informe Ejecutivo II Encuesta nacional de condiciones de seguridad y salud en el trabajo en el sistema general de riesgos. Bogotá: Ministerio de Trabajo de Colombia.

Ministerio de Trabajo. (2015). Decreto 1072 de 2015, Decreto Único Reglamentario del Sector Trabajo. Bogotá: Ministerio de Trabajo.

Ministerio de Trabajo. (2016). Indicadores del sistema general de riesgos laborales cifras 2011-2015. Bogotá: Dirección de Riesgos Laborales.

Molano, J. H., y Arévalo, N. (Abril-junio de 2013). De la Salud Ocupacional a la Gestión de la Seguridad y Salud en el Trabajo: más que semántica, una transformación del Sistema General de Riesgos Laborales. Revista Innovar Journal, 23(48), 30.

Nácher, J. (2002). Centro Internacional de Investigación. Información sobre Economía Pública Social y Cooperativa. Teoría de la participación: sugerencias analiticas. Valencia, España.

OIT. (2001). Directrices relativas a los sistemas de gestión de la seguridad y salud en el trabajo. ILO-OHS. Oficina Internacional de Ginebra. Ginebra: OIT, Organización Internacional del Trabajo.

OIT. (2011). Sistema de Gestión de la SST: una herramienta para la mejora continua. Recuperado de http:// 
Metodología para fortalecer la participación del personal en el Sistema de Gestión de Seguridad y Salud en el Trabajo en una empresa del sector aeronáutico

www.ilo.org/wcmsp5/groups/public/@ed_protect/@protrav/@safework/documents/publication/ wcms_154127.pdf.

Plan Nacional de Salud Ocupacional. (2013). Dirección General de Riesgos Laborales y el Comité Nacional de Salud Ocupacional del Ministerio del Trabajo. Bogotá. Recuperado de https://www.oiss.org/IMG/pdf/ PlanNacionalDeSeguridadySaludEnElTrabajo.pdf

Rodríguez, M. A. (2009). La participación de los trabajadores del centro de cómputo de la Universidad $\mathrm{Na}$ cional de Colombia, sede Bogotá, en los programas de salud ocupacional. Bogotá.

Rodríguez, Y. (2012). Estrategias para el mejoramiento de la gestión de la salud y seguridad en el trabajo frente a las formas de vinculación en plantas de un grupo empresarial del sector industrial de Bogotá D.C. Tesis de maestría, Universidad Nacional de Colombia, Bogotá, Colombia.
Rodríguez, Y. L., y Molano, J. H. (2012). Adaptación de una herramienta para la evaluación de la gestión de la seguridad y salud en el trabajo. El hombre y la máquina, (40), 8. Bogotá.

Secretaría General de la Alcaldía Mayor de Bogotá. (1994). Por el cual se determina la organización y administración del Sistema General de Riesgos Profesionales. [Decreto 1295 de 1994]. DO: 41.405.

Secretaría General de la Alcaldía Mayor de Bogotá. (2014). Por el cual se dictan disposiciones para la implementación del Sistema de Gestión de la Seguridad y Salud en el Trabajo (SG-SST). [Decreto 1443 de 2014]. DO: 49.229.

Secretaría General de la Alcaldía Mayor de Bogotá. (2017). Por medio del cual se modifica el artículo 2.2.4.6.37. del Decreto número 1072 de 2015, Decreto Único Reglamentario del Sector Trabajo, sobre la transición para la implementación del Sistema de Gestión de la Seguridad y Salud en el Trabajo (SG-SST). [Decreto 052 de 2017]. DO: 50.114. 